\title{
Absence of cytotoxic and inflammatory effects following in vitro exposure of chondrogenically-differentiated human mesenchymal stem cells to adenosine, lidocaine and $\mathrm{Mg}^{2+}$ solution
}

Andrew McCutchan ${ }^{1}$, Geoffrey P. Dobson², Natalie Stewart ${ }^{1}$, Hayley L. Letson², Andrea L. Grant ${ }^{3}$, Ivana-Aleksandra Jovanovic ${ }^{3}$, Kaushik Hazratwala ${ }^{2,3}$, Matthew Wilkinson ${ }^{2,3}$, Peter McEwen ${ }^{2,3}$ and Jodie Morris M $^{2,3^{*}}$ (D)

\begin{abstract}
Background: ALM solution, a combination of adenosine, lidocaine and $\mathrm{Mg}^{2+}$, is an emerging small volume therapy that has been shown to prevent and correct coagulopathy and surgery-related inflammation in preclinical models, though its application in orthopaedic surgery is yet to be demonstrated. The effect of ALM solution on chondrocytes is unknown. The aim of this preliminary study was to investigate the effect of ALM solution on viability and inflammatory responses of chondrogenically-differentiated human bone marrow-derived mesenchymal stem cells (chondro-MSC), in vitro.

Methods: Chondro-MSC were exposed to media only, saline $(0.9 \% \mathrm{NaCl}$ or $1.3 \% \mathrm{NaCl})$ only, or saline containing ALM (1 mM adenosine, $3 \mathrm{mM}$ lidocaine, $2.5 \mathrm{mM} \mathrm{Mg}^{2+}$ ) or tranexamic acid (TXA, $100 \mathrm{mg} / \mathrm{ml}$ ) for 1 or $4 \mathrm{~h}$. Responses to ALM solutions containing higher lidocaine concentrations were also compared. Chondrocyte viability was determined using WST-8 colorimetric assays and inflammatory cytokine (TNF-a, IL-1 $\beta, I L-8$ ) and matrix metalloproteinases (MMP-3, MMP-12, MMP-13) concentrations using multiplex bead arrays.

Results: The viability of chondro-MSC was significantly greater after $1 \mathrm{~h}$ treatment with ALM compared to saline ( $96.2 \pm 7.9$ versus $75.6 \pm 7.3 \%$ ). Extension of exposure times to $4 \mathrm{~h}$ had no significant adverse effect on cell viability after treatment with ALM $(1 \mathrm{~h}, 85.4 \pm 5.6 \vee 4 \mathrm{~h}, 74.0 \pm 15.2 \%)$. Cytotoxicity was evident following exposure to solutions containing lidocaine concentrations greater than $30 \mathrm{mM}$. There were no significant differences in viability $(80 \pm 5.4 \vee 57.3 \pm 16.2 \%)$ or secretion of IL-8 $(60 \pm 20 \vee 160 \pm 50 \mathrm{pg} / \mathrm{ml}), \mathrm{MMP}-3(0.95 \pm 0.6 \vee 3.4 \pm 1.6 \mathrm{ng} / \mathrm{ml})$, and MMP-13 $(4.2 \pm 2.4 \vee 9.2 \pm 4.3 \mathrm{ng} / \mathrm{ml})$ in chondro-MSC exposed to saline, ALM or TXA.

Conclusions: Short-term, in vitro exposure to clinically-relevant concentrations of ALM solution had no adverse inflammatory or chondrotoxic effects on human chondro-MSC, with responses comparable to saline and TXA. These findings provide support for continued evaluation of ALM solution as a possible therapeutic to improve outcomes following orthopaedic procedures.
\end{abstract}

Keywords: Chondro-MSC, Chondrocyte, ALM, Tranexamic acid, Viability, Inflammation

\footnotetext{
*Correspondence: jodie.morris@oriql.com.au

${ }^{2}$ Heart, Trauma and Sepsis Research Laboratory, College of Medicine and

Dentistry, James Cook University, Townsville, Australia

${ }^{3}$ The Orthopaedic Research Institute of Queensland, 7 Turner St, Pimlico,

Townsville Q 4812, Australia

Full list of author information is available at the end of the article
} 


\section{Background}

An ageing population and rise in prevalence of comorbid chronic disease is projected to drive a continued global increase in demand for elective arthroscopy and arthroplasty procedures (Inacio et al. 2017a, b, Holtedahl et al. 2018). As with any surgery, there are a number of potential complications following orthopaedic surgical procedures including local bleeding, coagulopathy, inflammation and tissue trauma (Cheuy et al. 2017, Friberger Pajalic et al. 2018). While inflammation and immune modulation are essential for tissue repair, sustained or overexpression of these responses can lead to further bleeding, joint damage, infection and arthrofibrosis (Eming et al. 2007, Dobson 2015, Järvinen and Guyatt 2016, Werthel et al. 2017).

The anti-fibrinolytic tranexamic acid (TXA) has become increasingly popular in orthopaedic procedures to improve visualization and reduce some of these complications (Karaaslan et al. 2015, Demos et al. 2017, Abdel et al. 2018). However, debate continues regarding the effect of TXA on articular cartilage and post-operative inflammation (Jang et al. 2014, Goyal et al. 2017, Fillingham et al. 2018, Grant et al. 2018, Moskal and Capps 2018, Parker et al. 2018). A combination of adenosine, lidocaine and $\mathrm{Mg}^{2+}$ (ALM solution) is an emerging small volume therapy that has been shown to prevent and correct coagulopathy and surgery-related inflammation, and reduce the trauma of surgery in several preclinical models (Dobson and Letson 2016). In contrast to TXA, which acts as a downstream anti-fibrinolytic (Reed and Woolley 2015, Dobson et al. 2018), ALM appears to correct coagulopathy upstream at the thrombomodulin complex located on the endothelium (Dobson et al. 2015, Dobson and Letson 2016). In addition, ALM has been shown to be a potent anti-inflammatory following hemorrhagic shock, traumatic brain injury, abdominal surgery and polymicrobial sepsis (Granfeldt et al. 2014, Griffin et al. 2014, Letson and Dobson 2015, Griffin et al. 2016, Davenport et al. 2017, Letson and Dobson 2017a, b Letson and Dobson 2018a, b). The application of ALM solution in orthopaedic surgical procedures is yet to be described. However, based on preclinical models of major surgery (Dobson and Letson 2016, Davenport et al. 2017), ALM solution has the potential to correct coagulopathy and reduce tissue-damaging inflammatory responses following joint injury and during orthopaedic procedures. As a precursor to evaluating ALM solution in orthopaedic models, preliminary in vitro studies are necessary to determine if clinically-relevant concentrations of ALM solution exert potentially adverse effects on human chondrocytes. Due to difficulties associated with isolating and maintaining primary human chondrocytes cultures at sufficient cell densities for in vitro characterisation assays, chondrogenically-differentiated human bone marrow-derived mesenchymal stem cells
(chondro-MSC) were used as a substitute for these proof-of-concept studies. Our aim was to investigate the effect of ALM solution on viability and inflammatory responses of chondro-MSC, in vitro. We hypothesised that ALM solution would show no cytotoxic or stimulatory effect on chondro-MSC, and would be comparable to saline and TXA.

\section{Methods}

Approvals

Informed consent was obtained prospectively from all participants, and the study was approved by the Institutional Human Research Ethics Committee (HREC/ 15/QTHS/39). The research undertaken strictly adhered to the Code of Ethics (Declaration of Helsinki) of the World Medical Association for trials involving humans.

\section{Cell isolation, culture conditions and in vitro chrondrogenic differentiation}

Ten patients ( 8 male, 2 females; age range 20-66 years) undergoing a bone marrow biopsy procedure as part of a routine assessment of their remission status for a haematological condition were recruited to participate in the study following informed consent. The buffy coat from an aspirated bone marrow sample $(1 \mathrm{ml})$ was isolated from each patient and washed in HBSS. Nucleated cells $\left(2 \times 10^{7}\right)$ were plated in $75 \mathrm{~cm}^{2}$ culture flasks (T75) with Expansion media (Dulbecco Modified Eagle Medium, DMEM + GlutaMAX, Thermo Fisher Scientific, Scoresby, VIC) supplemented with foetal bovine serum (10\%, Sigma-Aldrich, Castle Hill, NSW), human fibroblast growth factor $(2 \mathrm{ng} / \mathrm{ml}$, Miltenyi Biotec, Macquarie Park, NSW), and penicillin/ streptomycin (1\%, Thermo Fisher Scientific), and cultured for $24 \mathrm{~h}$ at $37^{\circ} \mathrm{C}$ in a humidified atmosphere of $5 \% \mathrm{CO}_{2}$. Non-adherent cells were removed after $24 \mathrm{~h}$ culture, with further media changes performed three times a week (Mareschi et al. 2012). To promote an even distribution of MSC, at day 14 the cells were detached with TrypLE ${ }^{\mathrm{mm}}$ Express (Thermo Fisher Scientific), washed and transferred to new flasks, with secondary cultures reaching $80-90 \%$ confluence within 7 days of subculture. To minimise any loss of differentiation potential, MSC were not cultured beyond the second passage.

In vitro chondrogenesis was performed according to published protocols (Solchaga et al. 2011). Expanded MSC were trypsinised, washed and $7.5 \times 10^{5}$ cells transferred to new T75 flasks. Chondrogenic differentiation was induced by treatment with STEMPRO ${ }^{\mathrm{m}}$ Chondrogenesis Differentiation medium (Thermo Fisher Scientific) (Kalamegam et al. 2016). Monolayers were incubated at $37^{\circ} \mathrm{C}$ in $5 \% \mathrm{CO}_{2}$ with differentiation media 
changes performed three times a week. After 7 days, the monolayer was observed microscopically to confirm chondrocyte morphology. Differentiation was regarded as $>90 \%$ conversion of a spindle-shaped, fibroblastic MSC morphology to a rounded, polygonal morphology consistent with chondrocyte monolayers (Fig. 1a, b). Chondro-MSC were not cultured beyond first passage to avoid de-differentiation. Following visual confirmation, chondro-MSC were trypsinised, washed and counted. Chondro-MSC were transferred to the wells of a 96-well plate at high cell densities $\left(>5 \times 10^{3}\right)$ to avoid de-differentiation and were subsequently used for assessment of viability and cytokine production in response to ALM and TXA exposure as described below.

\section{Histology}

To confirm chondrogenic differentiation of MSC using the culture conditions described above, $5 \times 10^{5}$ MSC were placed in the wells of a 24-well Transwell $^{\circ}$ plate (Sigma-Aldrich) and differentiation media was added, with media changes performed three times a week (Murdoch et al. 2009). After 21 days, the Transwell membrane was removed and fixed in $4 \%$ paraformaldehyde, processed histologically and paraffin-embedded sections stained with hematoxylin and eosin (H\&E), toluidine blue and Picro-Mallory trichrome. Chondrocyte aggregation and early stages of matrix structural organisation was in evident histologically. Sections stained with toluidine blue demonstrated the presence of negatively charged proteoglycans, whilst blue colouration in trichrome-stained sections confirmed the deposition of a collagenous matrix between chondrocytes (Fig. 1c, d).

\section{Treatments}

Adenosine (A9251), Lidocaine- $\mathrm{HCl}$ (L5647), and $\mathrm{MgSO}_{4}$ (M7506) were purchased from Sigma-Aldrich. Chondrocytes were incubated 1) in media only (no Tx), 2) saline $(0.9 \% \mathrm{NaCl}$ or $1.3 \% \mathrm{NaCl})$ only, 3) adenosine $(1 \mathrm{mM})$ alone, lidocaine $(3 \mathrm{mM})$ alone, or $\mathrm{Mg}^{2+}(2.5 \mathrm{mM})$ alone, and 4) ALM solution combined (adenosine $1 \mathrm{mM}$, lidocaine $\left.3 \mathrm{mM}, \mathrm{Mg}^{2+} 2.5 \mathrm{mM}\right)$. Given that $1 \%(\sim 37 \mathrm{mM})$ and $2 \%(\sim 74 \mathrm{mM})$ lidocaine solutions are known to be toxic to chondrocytes (Karpie and Chu 2007, Baker et al. 2011), we also examined ALM with $30 \mathrm{mM}$ lidocaine $\left(\mathrm{AL}_{30} \mathrm{M}\right)$ and $60 \mathrm{mM}$ lidocaine $\left(\mathrm{AL}_{60} \mathrm{M}\right)$ as internal controls. In addition, ALM solution ( $0.9 \%$ saline) was compared to TXA $(100 \mathrm{mg} / \mathrm{ml}$ in $0.9 \%$ saline, Juno Pharmaceuticals, South Yarra, VIC). Since intra-articular doses of 1 to $3 \mathrm{~g}$ of TXA in $20 \mathrm{ml}$ saline $(50-150 \mathrm{mg} / \mathrm{ml})$ are used in our institution and by others (Ishida et al.
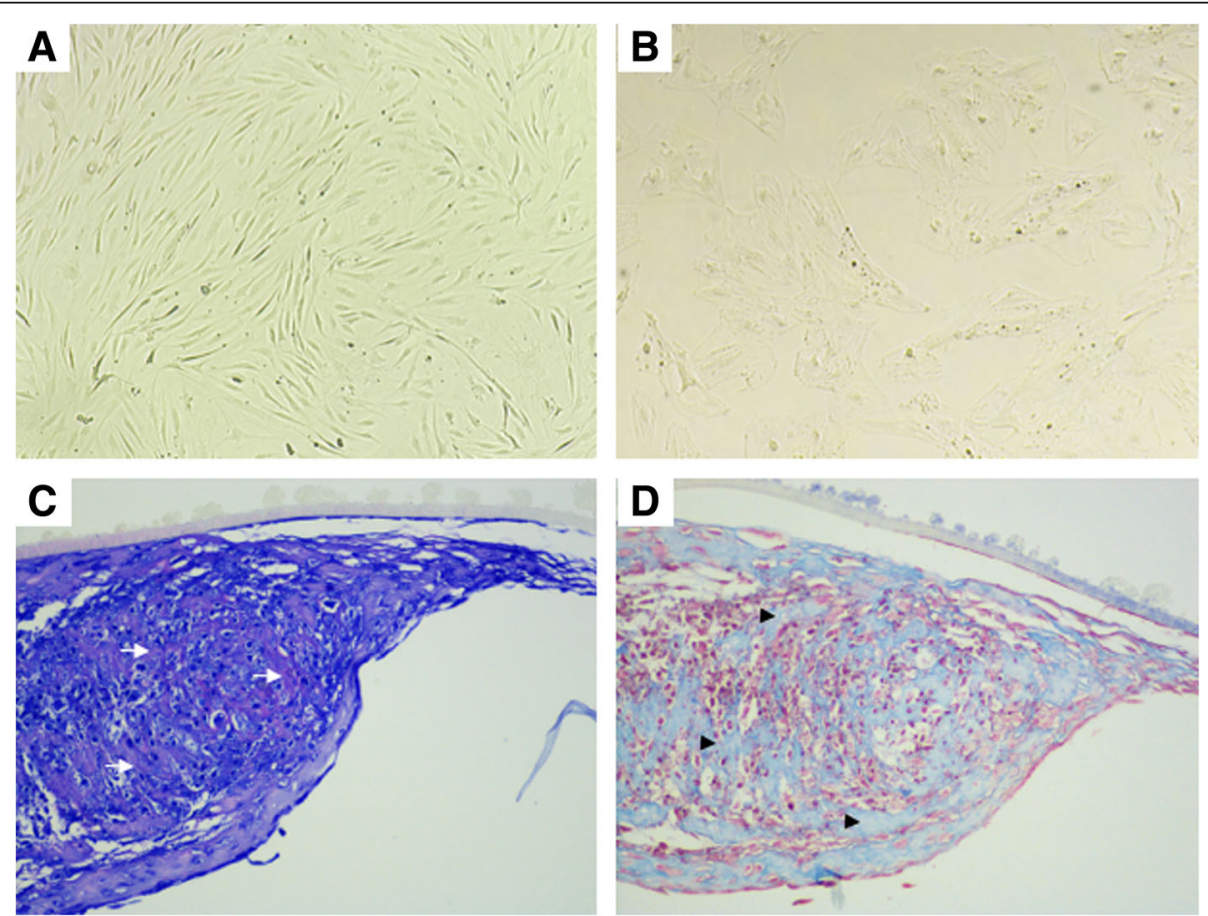

Fig. 1 Chondrogenic differentiation of human MSC. Cellular morphology changes were evident in human MSC (a) prior to, and (b) following culture in chondrogenic differentiation media for 7 days, with the transformation of spindle-shaped MSC to rounded, polygonal chondrocytes (magnification, 10x). Chondrogenic differentiation was confirmed histologically, with chondrocyte aggregation and early structural organisation of a collagenous matrix evident. Representative formalin-fixed, paraffin-embedded sections of chondro-MSC were stained with (c) toluidine blue to demonstrate sulphated glycosaminoglycans deposition (white arrow) or (d) Picro-Mallory trichrome stain highlighting deposition of collagen fibres (black arrowhead) within the extracellular matrix (magnification, 20x) 
2011, Tuttle et al. 2014), a concentration of $100 \mathrm{mg} / \mathrm{ml}$ TXA was selected for comparison to ALM solution in the current study. Chondro-MSC were exposed to treatments for 1 or $4 \mathrm{~h}\left(37^{\circ} \mathrm{C}, 5 \% \mathrm{CO}_{2}\right)$ prior to processing for assessment of viability or secreted inflammatory cytokines, chemokines and matrix metalloproteinases (MMP). Exposure times of 1 and $4 \mathrm{~h}$, were selected to reflect the mean surgical times associated with arthroplasty procedures (Weber et al. 2018). The longer exposure time reflects the $4 \mathrm{~h}$ ALM infusion times used in previous in vivo studies to confer protection in preclinical models of trauma, sepsis and surgery (Griffin et al. 2014, Griffin et al. 2016, Letson and Dobson, 2017a, b).

\section{Viability assay}

Chondrocyte viability was assessed using a WST-8 colorimetric assay, according to manufacturers' protocol (Cell Count Kit-8, Sigma Aldrich). The WST-8 assay assesses mitochondrial ability to reduce WST-8 into water-soluble tetrazolium salt. Briefly, chondro-MSC were enzymatically lifted from flasks and dispensed into a 96-well plate at $5 \times 10^{3}$ cells/well. After incubation for $24 \mathrm{~h}$ to allow attachment, ALM or individual components were added to appropriate wells for 1 or $4 \mathrm{~h}\left(37^{\circ}\right.$ C, $5 \% \mathrm{CO}_{2}$ ). After treatment, the medium was removed, fresh culture media added and plates incubated for a further $18 \mathrm{~h}\left(37^{\circ} \mathrm{C}, 5 \% \mathrm{CO}_{2}\right)$. WST-8 $(10 \mu \mathrm{l})$ was added and plates incubated for a further $4 \mathrm{~h}\left(37^{\circ} \mathrm{C}, 5 \% \mathrm{CO}_{2}\right)$. Absorbance was measured at $450 \mathrm{~nm}$, with a reference of $650 \mathrm{~nm}$ using a Multiskan EX microplate reader (Thermo Fisher Scientific). Cell viability was calculated using the ratio of the absorbance of treated (saline, ALM or individual components, TXA) cells to the absorbance of untreated (culture media only) cells, and data were expressed as percentages.

\section{Cytokine, chemokine and MMP analysis}

In a parallel set of experiments, the secretion of inflammatory cytokines, chemokines and MMP from chondro-MSC was compared in ALM- and TXA-treated cultures. Briefly, expanded MSC were enzymatically lifted from flasks and dispensed into a 96 -well plate $(5 \times$ $10^{4}$ cells/well). After overnight incubation, expansion media was replaced with chondrogenic differentiation media, and cultures incubated for 7 days with differentiation media changes performed three times. Saline only (0.9\%), ALM, or TXA solutions were added to appropriate wells for $1 \mathrm{~h}$. Recombinant human IL-1 $\beta$ was purchased from Sigma Aldrich (19401) and included in each experiment as a positive control $(5 \mathrm{ng} / \mathrm{ml}$; Forsyth et al. 2005). Chondro-MSC were washed, media replaced and cells incubated for a further $24 \mathrm{~h}$ prior to removal of cell-free supernatants from untreated and treated wells. Milliplex ${ }^{\circ}$ Human Cytokine/Chemokine (HCYTOMAG-60
K, Lot \#2929279) and MMP Magnetic Bead Panel 1 (HMMP1MAG-55 K, Lot \#10101, Abacus ALS, Meadowbrook, Queensland) in combination with the Magpix ${ }^{\circ}$ analyser (Luminex Corporation, Austin, Texas, USA) were used to measure levels of interleukin (IL)-8, IL-1 $\beta$, tumor necrosis factor alpha (TNF- $\alpha$ ), MMP-3, MMP-12 and MMP-13 in chondro-MSC culture supernatants. Assays were carried out according to manufacturer's instructions with samples measured in duplicate.

\section{Statistics}

GraphPad Prism 7 was used for all data analysis. Data normality was assessed using Shapiro-Wilks test, with Levene's test used to determine equality of variances. Viability between groups were compared using t-tests, univariant variance analysis (ANOVA, Tukey post-hoc analysis) or two-way ANOVA (Tukey post-hoc analysis) where appropriate. MILLIPLEX Analyst 5.1 software (Luminex Corporation, Austin, Texas, USA), which analyses data with a 5-parametric logistic weighted curve fit, was used to determine cytokine and MMP concentrations. Cytokine and MMP concentrations between groups were compared using Kruskal-Wallis test with Dunn's post-hoc analysis. All values are expressed as mean \pm standard error of the mean (SEM) with significance set at $P<0.05$.

\section{Results}

\section{ALM improves chondrocyte viability}

The viability of chondro-MSC treated with ALM solution for $1 \mathrm{~h}$ was significantly greater than cultures exposed to normal saline $(96.2 \pm 7.9 \% \vee 75.6 \pm 7.3 \%, P$ $=.045$, Fig. 2a). In contrast, chondrotoxicity was demonstrated by significantly reduced viability in cultures exposed to ALM solution where lidocaine concentrations were increased to $30 \mathrm{mM}(44.5 \pm 4.6 \%)$ or $60 \mathrm{mM}$ $(5.8 \pm 3.1 \%, P<0.001$, Fig. $2 b)$. There was no difference in the viability of ALM-treated chondro-MSC between 1 and $4 \mathrm{~h}$ of exposure $(85.4 \pm 5.6 \% \vee 74 \pm 15.2 \% . P=.214$, Fig. 2c).

Exposure of chondro-MSC to adenosine alone (1 $\mathrm{mM})$ or lidocaine alone $(3 \mathrm{mM})$ resulted in viability that was comparable to cultures exposed to saline vehicle (saline, $86.9 \pm 4.9 \%$; adenosine, $87.6 \pm 5.6 \%$; lidocaine $94.5 \pm 7.1 \% ; P=.93)$. In separate experiments, viability was significantly higher in chondro-MSC cultures exposed to normal saline with $\mathrm{Mg}^{2+}$ compared to saline alone $(108 \pm 6.8 \% \vee 79.3 \pm 7.6 \%, P=.043$, Fig. 2e). Viability was comparable between $\mathrm{Mg}^{2}$-supplemented saline and ALM-treated chondro-MSC cultures $(108 \pm 6.8 \% \vee 97.6 \pm 9.4 \%$, Fig. 2 e) .

Chondroprotection has previously been demonstrated in response to increased osmolarity in bovine cartilage explant cultures ( $480 \mathrm{mOsm}, \sim 1.3 \%$ saline) 
A

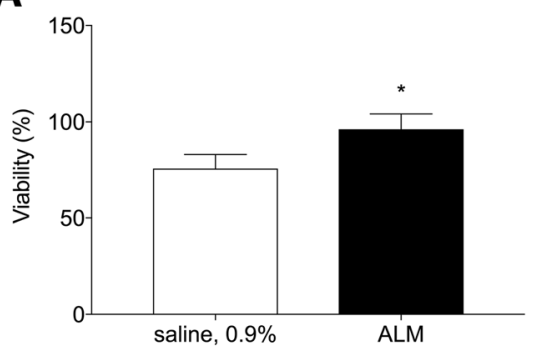

C

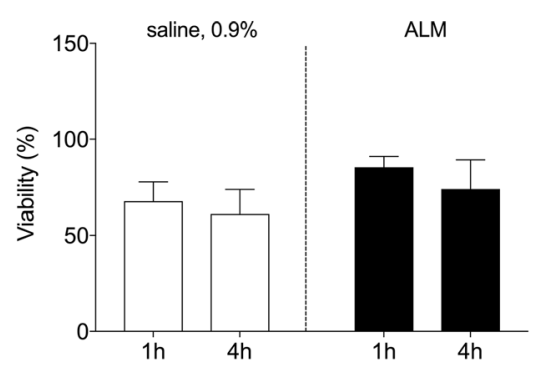

E

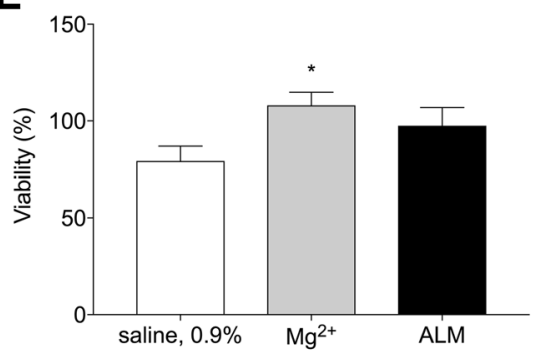

B

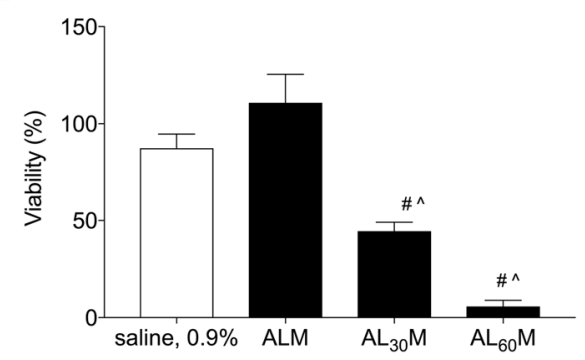

D

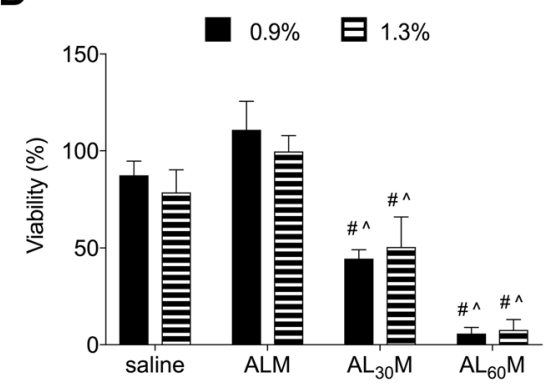

$\mathbf{F}$

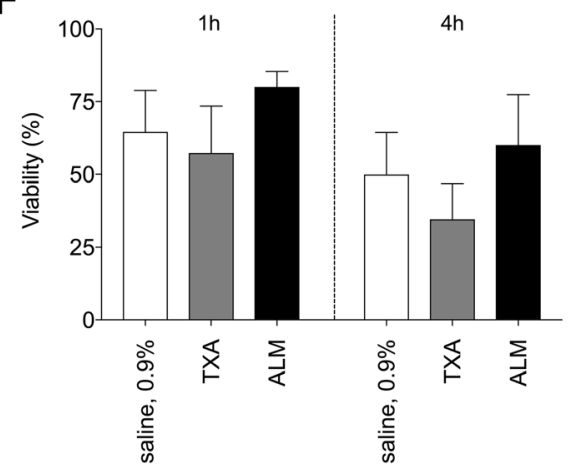

Fig. 2 Effect of saline, ALM and TXA solutions on chondro-MSC viability, where cell viability is defined as the mean absorbance of treated (saline, ALM, TXA) cells normalised to the mean absorbance of untreated (culture media only) cells. a Compared to $0.9 \%$ saline, viability was significantly greater in cultures exposed to ALM solution for $1 \mathrm{~h}(P=.045, n=13)$. $\mathbf{b}$ Increasing lidocaine concentrations within the ALM preparation to $30 \mathrm{mM}\left(\mathrm{AL}_{30} \mathrm{M}\right)$ and $60 \mathrm{mM}\left(\mathrm{AL}_{60} \mathrm{M}\right)$ resulted in significantly reduced viability $(P<.001, n=6$, \# compared to saline; $\wedge$ compared to ALM). c There were no significant differences in chondrocyte viability at 1 and $4 \mathrm{~h}$ exposure of chondrocytes to ALM solution. $\mathbf{d}$ No significant differences were observed between $0.9 \%$ and $1.3 \%$ saline $(n=6)$. e Compared to normal saline $(0.9 \%)$, addition of $\mathrm{Mg}^{2+}$ significantly improved cell viability $(P=.043, n=11)$. $\mathbf{f}$ No significant differences were observed in the viability of cultures exposed to ALM solution or TXA for $1(P=.47)$ or $4 \mathrm{~h}(P=.49 ; n=5)$. Data is expressed as mean \pm S.E.M. ALM, adenosine, lidocaine, and magnesium; $\mathrm{MgSO}_{4}$, magnesium sulphate; MSC, mesenchymal stem cells; TXA, tranexamic acid

(Amin et al. 2010). To investigate whether chondrocyte viability was affected by saline solution osmolarity in the current study, chondro-MSC were cultured in the presence of $0.9 \%$ or $1.3 \%$ saline only, ALM in $0.9 \%$ saline or ALM in $1.3 \%$ saline. No significant differences were observed in the viability of chondro-MSC exposed to either $0.9 \%$ or $1.3 \%$ saline $(87.4 \pm 7.3 \% \quad v 78.6 \pm 11.7 \%, P=.894)$. Similarly, the viability of chondro-MSC exposed to ALM in $0.9 \%$ saline was comparable to cultures exposed to ALM in $1.3 \%$ saline $(P=.667$, Fig. $2 \mathrm{~d})$.

\section{Effects of ALM and TXA on chondrocyte viability and inflammatory response}

No significant differences were observed in the viability of chondro-MSC exposed to ALM or TXA for $1 \mathrm{~h}$ (80 \pm $5.4 \% \vee 57.3 \pm 16.2 \%, P=.47)$ or $4 \mathrm{~h}(60.1 \pm 17.3 \% \vee 34.6$ $\pm 12.3 \%, P=.49$, Fig. 2 f) .

Cell-free culture supernatants were assessed for inflammatory cytokine and chemokines and MMP concentrations following exposure to ALM or TXA for $1 \mathrm{~h}$ (Fig. 3). Levels of IL-1 $\beta$, TNF- $\alpha$ and MMP-12 were below the limit of detection for each treatment group, despite measurable 
A

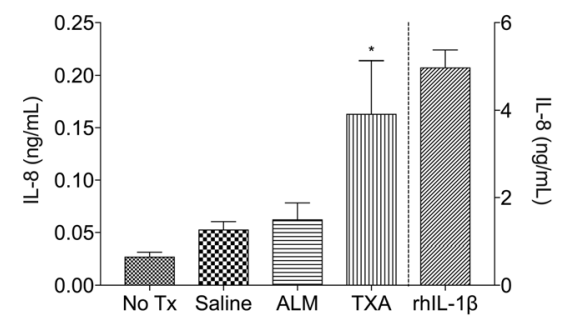

C

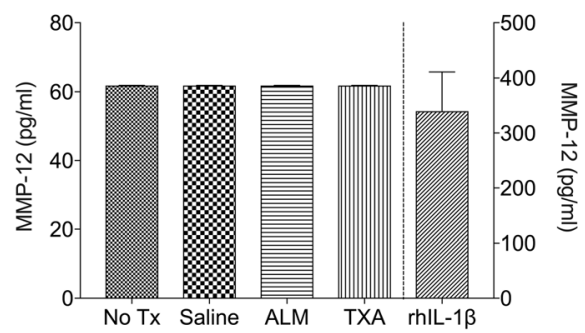

B

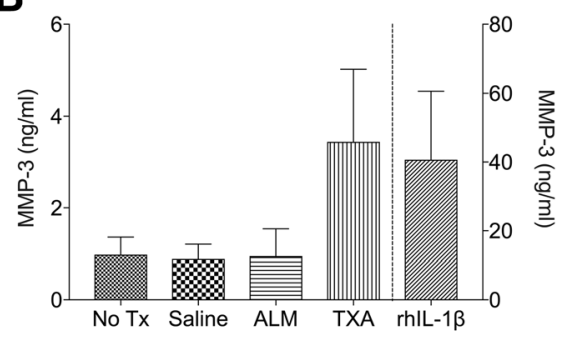

D

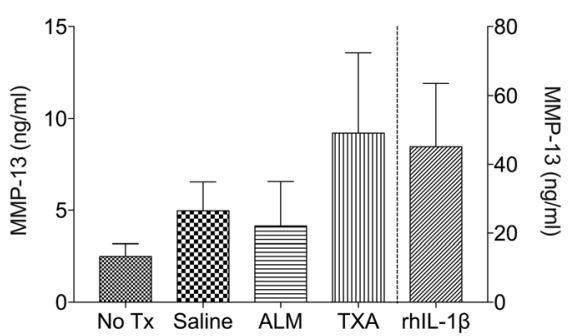

Fig. 3 Concentration of (a) IL-8, (b) MMP-3, (c) MMP-12 and (d) MMP-13 in chondro-MSC cultures left untreated (culture media only) or exposed to $0.9 \%$ saline, ALM, TXA or recombinant human IL-1 $\beta$. Data is expressed as mean \pm S.E.M. ${ }^{*} P<0.05$ compared to no treatment. ALM, adenosine, lidocaine, and magnesium; IL, interleukin; MMP, matrix metalloproteinase; MSC, mesenchymal stem cells; TXA, tranexamic acid

levels in rhIL-1 $\beta$-treated chondro-MSC cultures (IL-1 $\beta$ $3.0 \pm 0.2 \mathrm{pg} / \mathrm{ml}$, TNF- $\alpha 2.8 \pm 0.5 \mathrm{pg} / \mathrm{ml}, \mathrm{MMP}-12,340 \pm$ $71.2 \mathrm{pg} / \mathrm{ml})$. In contrast, secretion of IL-8 was significantly increased in chondro-MSC cultures exposed to TXA ( $P$ $=.044$ ) compared to untreated (culture media only) cells (Fig. 3a). Levels of MMP-3 and MMP-13 were comparable for between treatment groups, however greater variability was observed in the concentrations of these MMPs in cultures exposed to TXA (Fig. 3b).

\section{Discussion}

We report the absence of cytotoxic and inflammatory responses following short-term, in vitro exposure of human chondro-MSC to ALM solution. ALM solution is an emerging small-volume therapy containing a combination of adenosine, lidocaine and magnesium (Dobson and Letson 2016). At high concentrations, ALM solution arrests the heart and is currently in clinical use as a method for polarizing cardioplegia (Djabir et al. 2013). At 10-fold lower 'non-arrest' concentrations, ALM solution has been shown to correct coagulopathy, improve cardiac function, and reduce immune-inflammatory activation in response to sterile surgery and trauma in preclinical models (Granfeldt et al. 2014, Dobson et al. 2015, Letson and Dobson 2015, Davenport et al. 2017, Letson and Dobson 2017a, b, Letson and Dobson 2018a, b).

\section{ALM appears to be safe at low lidocaine concentrations} The present study found that ALM solution (3 $\mathrm{mM}$ or $0.08 \%$ lidocaine) significantly improved chondrocyte viability by $30 \%$ compared to normal saline. No benefit was found using a higher $1.3 \%$ hypertonic salt solution, which some groups have reported is more chondroprotective than normal saline (Amin et al. 2010). Consistent with the findings of previous studies using human chondrocytes (Karpie and Chu 2007, Baker et al. 2011), lidocaine at higher concentrations (30 and $60 \mathrm{mM}$ ) was toxic to chondro-MSC in the current study, with cell viability decreasing by $65 \%$ and $95 \%$, respectively. The mechanisms for lidocaine toxicity at $1 \%(37 \mathrm{mM})$ or $2 \%$ (74 mM) concentrations, and other local anesthetics (e.g. bupivacaine, ropivacaine, mepivacaine), appear to be associated with impairment of chondrocyte metabolism, which leads to cell death (Kreuz et al. 2018). Dragoo and colleagues (Dragoo et al. 2012) also showed that 1\% lidocaine in human monolayer chondrocyte cultures was associated a reduction of extracellular matrix molecules, glycosaminoglycan, and collagen content compared to controls after $3 \mathrm{~h}$. Interestingly, Baker et al. (2011) showed that lidocaine toxicity could be attenuated with the addition of magnesium. In the present study, we found the addition of magnesium sulphate to normal saline led to $10 \%$ higher cell viability. The significance of using ALM solution compared to $\mathrm{Mg}^{2+}$ alone will be discussed in Potential Clinical Relevance section (see below).

\section{Chondrocyte viability comparable for ALM and TXA solution}

Using a clinically relevant, intra-articular dose of TXA (100 mg/ml; Ishida et al. 2011, Tuttle et al. 2014), our study found no significant difference between normal 
saline, ALM and TXA after $1 \mathrm{~h}$ exposure, although mean chondro-MSC viability in TXA-exposed cultures was reduced by $15 \%$ and $29 \%$ compared to normal saline and ALM solution, respectively. Parker et al. (2018) recently demonstrated significant dose-response chondrotoxicity of TXA toward human chondrocytes using lower concentrations of TXA $(40 \mathrm{mg} / \mathrm{ml})$, with a $58 \%$ loss of viability in monolayers, and approximately $30 \%$ decrease in hydrogel-encapsulated 3D human chondrocyte cultures following $3 \mathrm{~h}$ exposure. Parker et al. (2018) and more recently, Ambra et al. (2017) recommended that intraarticular administration of TXA in orthopaedic surgery may be safe at TXA concentrations below 20 $\mathrm{mg} / \mathrm{ml}$ (Parker et al. 2018). Notwithstanding these studies, standardised protocols for intra-articular use of TXA are lacking and debate continues regarding potential detrimental effects following its use in arthroscopy and other orthopaedic procedures where native articular cartilage remains (Jang et al. 2014, Goyal et al. 2017, Fillingham et al. 2018, Grant et al. 2018, Moskal and Capps 2018, Parker et al. 2018). Further in vivo studies are required to evaluate potential underlying dose-dependent mechanisms of TXA chondrotoxicity.

\section{Absence of inflammatory activation of chondrocytes exposed to ALM}

Another interesting finding in our study was that ALM solution did not activate secretion of inflammatory mediators known to be involved in cartilage breakdown and joint deterioration (Chen et al. 2014, Pap and Korb-Pap 2015, Takahashi et al. 2015, Minguzzi et al. 2018) from human chondro-MSC cultures, with responses comparable to those exposed to normal saline (Fig. 3). No statistically significant difference was observed in inflammatory mediator concentrations between cultures exposed to either saline, ALM or TXA, although levels of IL-8 were significantly higher in chondro-MSC cultures treated with TXA compared to untreated cultures. IL-8 is expressed at increased levels in human osteoarthritic chondrocytes (Takahashi et al. 2015, Minguzzi et al. 2018) and is associated with: 1) production of IL-1 $\beta$, IL- 6 , and TNF- $\alpha, 2)$ the induction of chondrocyte hypertrophy and differentiation, 2) the release of MMP-13, and 3) neutrophil-mediated inflammation and cartilage breakdown from generation of reactive oxygen metabolites (Lotz et al. 1992, Takahashi et al. 2015). It is noteworthy that TXA administration in patients undergoing TKA was recently demonstrated to lead to significant increases in plasma inflammatory cytokines during and after surgery (Grant et al. 2018).

\section{Potential clinical relevance}

During orthopaedic surgical procedures, surgery-induced damage within joint tissues can activate chondrocytes in articular cartilage (Schulze-Tanzil 2009, Dobson 2015).
Chondrocyte activation leads to increased production of pro-inflammatory cytokines such as IL- $1 \beta$ and TNF- $\alpha$ which, in the absence of homeostatic regulation, drive an inflammatory cascade that promotes upregulation of cartilage-degrading proteinases and chondrocyte apoptosis (Schulze-Tanzil 2009, Pap and Korb-Pap 2015, Minguzzi et al. 2018). Strategies to reduce secondary injury progression are actively being sought to improve regulation of the postoperative healing process and patient outcomes. Small-volume bolus and infusion of ALM solution has been shown to improve survival and prevent secondary injury progression in a number of different trauma states in small and large animal models of hemorrhagic shock (Granfeldt et al. 2014, Dobson and Letson 2016, Letson and Dobson 2017a, b), cardiac arrest (Djabir et al. 2013), polymicrobial sepsis (Griffin et al. 2014, Griffin et al. 2016), abdominal surgery (Davenport et al. 2017), and more recently traumatic brain injury (Letson and Dobson 2018a, b). The addition of magnesium to normal saline was found to increase chondro-MSC viability in the current study and is consistent with in vitro chondroprotective properties described by others (Baker et al. 2011). The analgesic properties of magnesium may be due to its ability to block NMDA receptors and potentially through inhibitory effects on calcium and potassium channels, thus augmenting neural transmission (Srebro et al. 2016). Magnesium has been evaluated as an alternative intra-articular analgesic in arthroscopic knee surgery conferring better pain relief than placebo (Bondok and Abd El-Hady 2006), and showing a synergistic effect when combined with bupivacaine (Devi et al. 2018). Adenosine has also been shown to play a key role in cartilage homeostasis (Mediero and Cronstein et al. 2013). Importantly, preclinical studies have shown the whole-body protective benefits of ALM solution cannot be achieved using each drug individually (Dobson and Letson 2016). It is envisaged that an ALM infusion or intra-articular bolus may benefit patients undergoing orthopaedic interventions, and other major surgery, by reducing surgical stress, preventing bleeding and promoting a more permissive environment that enhances tissue healing following major surgery (Dobson 2015, Davenport et al. 2017). The potential clinical application of ALM solution in orthopaedic surgery as a novel strategy for protecting native articular cartilage and improving patient outcomes is currently being investigated.

\section{Limitations}

A potential limitation of the present study may be the use of chondrogenically differentiated human bone marrow derived MSC, rather than human primary chondrocytes or cartilage explants. However, Kalamegam et al. 
(2016) have shown chondrogenic differentiation of human bone marrow MSC using similar methods and culture supplements, with chondrocyte-like cells acquiring a polygonal morphology and increased expression of type II collagen within 7 days of culture and continued deposition of cartilaginous matrix over a 21-day period. We also acknowledge that different substrate surfaces were used for viability and cytokine assays (polystyrene) and histology of disc cultures (porous polycarbonate membrane), though chondrogenesis of MSC has been demonstrated for both (Kalamegam et al. 2016, Murdoch et al. 2009). Our findings suggest, even with direct contact for up to $4 \mathrm{~h}$, exposure to ALM solution had no adverse effect on chondrocyte-like cells in culture. However, in vitro studies cannot represent the complexities of the joint microenvironment and it is possible that the phenotypic responses we found in response to ALM solution may differ in vivo. Future studies that include more extensive investigation of inflammatory and oxidative stress responses of mature articular cartilage exposed to ALM solution in vivo are warranted and are the focus of current research.

\section{Conclusions}

We conclude that short-term exposure of human chondrocyte monolayers to ALM solution improved viability compared to saline alone. In addition, ALM did not stimulate secretion of key inflammatory mediators or matrix-degrading MMP that are believed to delay healing and promote secondary injury.

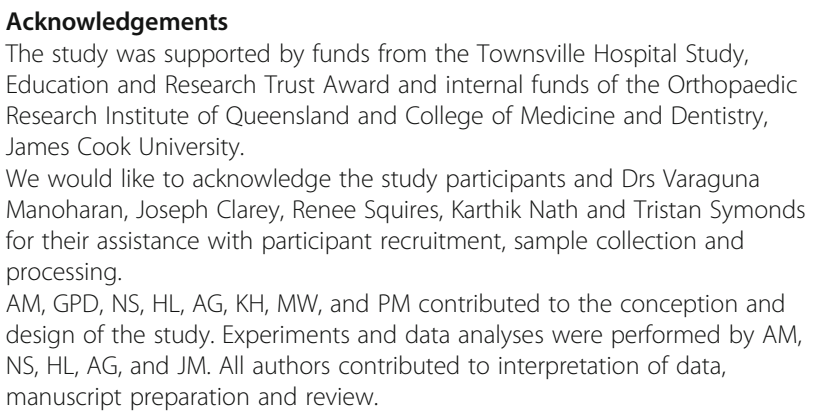

\section{Author's contributions}

$A M, G P D, N S, H L, A G, K H, M W$, and PM contributed to the conception and design of the study. Experiments and data analyses were performed by AM, $\mathrm{NS}, \mathrm{HL}, \mathrm{AG}$, and JM. All authors contributed to interpretation of data and manuscript preparation. All authors read and approved the final manuscript.

\section{Consent for publication}

Not applicable.

\section{Competing interests}

The authors declare they have no competing interests.

\section{Publisher's Note}

Springer Nature remains neutral with regard to jurisdictional claims in published maps and institutional affiliations.

\section{Author details}

'Department of Haematology and Bone Marrow Transplantation, Townsville Hospital, Townsville, Australia. ${ }^{2}$ Heart, Trauma and Sepsis Research Laboratory, College of Medicine and Dentistry, James Cook University, Townsville, Australia. ${ }^{3}$ The Orthopaedic Research Institute of Queensland, 7 Turner St, Pimlico, Townsville Q 4812, Australia.

Received: 9 January 2019 Accepted: 22 March 2019

Published online: 15 April 2019

\section{References}

Abdel MP, Chalmers BP, Taunton MJ, Pagnano MW, Trousdale RT, Sierra RJ, Lee YY, Boettner F, Su EP, Haas SB, Figgie MP, Mayman DJ (2018) Intravenous versus topical tranexamic acid in total knee arthroplasty: both effective in a randomized clinical trial of 640 patients. J Bone Joint Surg Am 100:10231029

Ambra LF, de Girolamo L, Niu W, Phan A, Spector M, Gomoll AH (2017) No effect of topical application of tranexamic acid on articular cartilage. Knee Surg Sports Traumatol Arthrosc. https://doi.org/10.1007/s007/s00167-017-4746-9

Amin AK, Huntley JS, Simpson AHRW, Hall AC (2010) Increasing the osmolarity of joint irrigation solutions may avoid injury to cartilage: a pilot study. Clin Orthop Relat Res 468:875-884

Baker JF, Byrne DP, Walsh PM, Mulhall KJ (2011) Human chondrocyte viability after treatment with local anesthetic and/or magnesium: results from an in vitro study. Arthroscopy 27:213-217

Bondok RS, Abd El-Hady AM (2006) Intra-articular magnesium is effective for postoperative analgesia in arthroscopic knee surgery. Br J Anaesth 97:389392

Chen JJ, Huang JF, Du WX, Tong PJ (2014) Expression and significance of MMP-3 in synovium of knee joint at different stage in osteoarthritis patients. Asian Pac J Trop Med 7:297-300

Cheuy VA, Foran JRH, Paxton RJ, Bade MJ, Zeni JA, Stevens-Lapsley JE (2017) Arthrofibrosis associated with total knee arthroplasty. J Arthroplast 32:26042611

Davenport L, Letson HL, Dobson GP (2017) Immune-inflammatory activation after a single laparotomy in a rat model: effect of adenosine, lidocaine and Mg2+ infusion to dampen the stress response. Innate Immun 23:482-494

Demos HA, Lin ZX, Barfield WR, Wilson SH, Robertson DC, Pellegrini VD Jr (2017) Process improvement project using tranexamic acid is cost-effective in reducing blood loss and transfusions after total hip and total knee arthroplasty. J Arthroplast 32:2375-2380

Devi MM, Gupta S, Amaravathi R, Udupa S, Hegde A, Ghosh S (2018) Comparison of efficacy of intra-articular plain bupivacaine and bupivacaine with adjuvants (dexmedetomidine and magnesium sulfate) for postoperative analgesia in arthroscopic knee surgeries: a prospective, randomised controlled trial. Anesth Essays Res 12:848-854

Djabir Y, Letson HL, Dobson GP (2013) Adenosine, lidocaine, and Mg2+ (ALM) increases survival and corrects coagulopathy after eight-minute asphyxial cardiac arrest in the rat. Shock 40:222-232

Dobson GP (2015) Addressing the global burden of trauma in major surgery. Front Surg. https://doi.org/10.3389/fsurg.2015.00043

Dobson GP, Doma K, Letson HL (2018) Clinical relevance of a p value: does tranexamic acid save lives after trauma or postpartum hemorrhage? J Trauma Acute Care Surg 84:532-536

Dobson GP, Letson HL (2016) Adenosine, lidocaine, and Mg2+ (ALM): from cardiac surgery to combat casualty care-teaching old drugs new tricks. J Trauma Acute Care Surg 80:135-145

Dobson GP, Letson HL, Sharma R, Sheppard FR, Cap AP (2015) Mechanisms of early trauma-induced coagulopathy: the clot thickens or not? I Trauma Acute Care Surg 79:301-309

Dragoo JL, Braun HJ, Kim HJ, Phan HD, Golish SR (2012) The in vitro chondrotoxicity of single-dose local anesthetics. Am J Sports Med 40:794799

Eming SA, Krieg T, Davidson JM (2007) Inflammation in wound repair: molecular and cellular mechanisms. J Investig Dermatol 127:514-525

Fillingham YA, Ramkumar DB, Jevsevar DS, Yates AJ, Shores P, Mullen K, Bini SA, Clarke HD, Schemitsch E, Johnson RL, Memtsoudis SG, Sayeed SA, Sah AP, Della Valle CJ (2018) The efficacy of tranexamic acid in total knee arthroplasty: a network meta-analysis. J Arthroplast. https://doi.org/10.1016/j. arth.2018.04.043 
Forsyth CB, Cole A, Murphy G, Bienias JL, Im H-J, Loeser RF Jr (2005) Increased matrix metalloproteinase-13 production with aging by human articular chondrocytes in responses to catabolic stimuli. J Gerontol A Biol Sci Med Sci 60:1118-1124

Friberger Pajalic K, Turkiewicz A, Englund M (2018) Update on the risks of complications after knee arthroscopy. BMC Musculoskelet Disord 19:179-179

Goyal N, Chen DB, Harris IA, Rowden NJ, Kirsh G, MacDessi SJ (2017) Intravenous vs intra-articular tranexamic acid in total knee arthroplasty: a randomized, double-blind trial. J Arthroplast 32:28-32

Granfeldt A, Letson HL, Hyldebrandt JA, Wang ER, Salcedo PA, Nielsen TK, Tønnesen E, Vinten-Johansen J, Dobson GP (2014) Small-volume 7.5\% NaCl adenosine, lidocaine, and Mg2+ has multiple benefits during hypotensive and blood resuscitation in the pig following severe blood loss: rat to pig translation. Crit Care Med 42:e329-e344

Grant AL, Letson HL, Morris JL, McEwen P, Hazratwala K, Wilkinson M, Dobson GP (2018) Tranexamic acid is associated with selective increase in inflammatory markers following total knee arthroplasty (TKA): a pilot study. J Orthop Surg Res 13:149

Griffin MJ, Letson HL, Dobson GP (2014) Adenosine, lidocaine and Mg2+ (ALM) induces a reversible hypotensive state, reduces lung edema and prevents coagulopathy in the rat model of polymicrobial sepsis. J Trauma Acute Care Surg 77:471-478

Griffin MJ, Letson HL, Dobson GP (2016) Small-volume adenosine, lidocaine and Mg2+ (ALM) 4 hour infusion leads to $88 \%$ survival after 6 days of experimental sepsis in the rat without antibiotics. Clin Vaccine Immunol. https://doi.org/10.1128/cvi.00390-16

Holtedahl R, Brox Jl, Aune AK, Nguyen D, Risberg MA, Tjomsland O (2018) Changes in the rate of publicly financed knee arthroscopies: an analysis of data from the Norwegian patient registry from 2012 to 2016. BMJ Open 8: e021199

Inacio MCS, Graves SE, Pratt NL, Roughead EE, Nemes S (2017a) Increase in total joint arthroplasty projected from 2014 to 2046 in Australia: a conservative local model with international implications. Clin Orthop Relat Res 475:21302137

Inacio MCS, Paxton EW, Graves SE, Namba RS, Nemes S (2017b) Projected increase in total knee arthroplasty in the United States - an alternative projection model. Osteoarthr Cartil 25:1797-1803

Ishida K, Tsumura N, Kitagawa A, Hamamura S, Fukuda K, Dogaki Y, Kubo S, Matsumoto T, Matsushita T, Chin T, Iguchi T, Kurosaka M, Kuroda R (2011) Intra-articular injection of tranexamic acid reduces not only blood loss but also knee joint swelling after total knee arthroplasty. Int Orthop 35:16391645

Jang B, Kao M, Bohm MT, Harris IA, Chen DB, MacDessi SJ (2014) Intra-articular injection of tranexamic acid to reduce blood loss after total knee arthroplasty. J Orthop Surg (Hong Kong) 22:146-149

Järvinen TLN, Guyatt GH (2016) Arthroscopic surgery for knee pain. BMJ 354: i3934. https://doi.org/10.1136/bmj.i3934

Kalamegam G, Abbas M, Gari M, Alsehli H, Kadam R, Alkaff M, Chaudhary A, AlQahtani M, Abuzenadah A, Kafienah W, Mobasheri A (2016) Pelleted bone marrow derived mesenchymal stem cells are better protected from the deleterious effects of arthroscopic heat shock. Front Physiol 7:180

Karaaslan F, Karaoğlu S, Yurdakul E (2015) Reducing intra-articular hemarthrosis after arthroscopic anterior cruciate ligament reconstruction by the administration of intravenous tranexamic acid:a prospective, randomized controlled trial. Am J Sports Med 43:2720-2726

Karpie JC, Chu CR (2007) Lidocaine exhibits dose- and time-dependent cytotoxic effects on bovine articular chondrocytes in vitro. Am J Sports Med 35:16211627

Kreuz PC, Steinwachs M, Angele P (2018) Single-dose local anesthetics exhibit a type-, dose-, and time-dependent chondrotoxic effect on chondrocytes and cartilage: a systematic review of the current literature. Knee Surg Sports Traumatol Arthrosc 26:819-830

Letson HL, Dobson GP (2015) Correction of acute traumatic coagulopathy with small-volume $7.5 \%$ nacl adenosine, lidocaine, and Mg2+ occurs within 5 minutes: a rotem analysis. J Trauma Acute Care Surg 78:773-783

Letson HL, Dobson GP (2017a) Adenosine, lidocaine and Mg2+ (ALM) fluid therapy attenuates systemic inflammation, platelet dysfunction and coagulopathy after non-compressible truncal hemorrhage. PLoS One 12 : e0188144
Letson HL, Dobson GP (2017b). 3\% NaCl adenosine, lidocaine, Mg2+ (ALM) bolus and 4 hours "drip" infusion reduces noncompressible hemorrhage by $60 \%$ in a rat model. J Trauma Acute Care Surg 82:1063-1072

Letson $\mathrm{HL}$, Dobson GP (2018a) $7.5 \% \mathrm{NaCl}$ resuscitation leads to abnormal clot fibrinolysis after severe hemorrhagic shock and its correction with $7.5 \% \mathrm{NaCl}$ adenosine, lidocaine, and mg(2+). J EmergTrauma Shock 11:15-24

Letson HL, Dobson GP (2018b) Adenosine, lidocaine, and Mg2+ (ALM) resuscitation fluid protects against experimental traumatic brain injury. J Trauma Acute Care Surg 84:908-916

Lotz M, Terkeltaub R, Villiger PM (1992) Cartilage and joint inflammation. Regulation of il-8 expression by human articular chondrocytes. J Immunol 148:466

Mareschi K, Rustichelli D, Calabrese R, Gunetti M, Sanavio F, Castiglia S, Risso A Ferrero I, Tarella C, Fagioli F (2012) Multipotent mesenchymal stromal stem cell expansion by plating whole bone marrow at a low cellular density: a more advantageous method for clinical use. Stem Cells Int 2012:e920581

Mediero A, Cronstein BN (2013) Adenosine and bone metabolism. Trends Endocrinol Metab 24:290-300

Minguzzi M, Cetrullo S, Adamo S, Silvestri Y, Flamigni F, Borzi RM (2018) Emerging players at the intersection of chondrocyte loss of maturational arrest, oxidative stress, senescence and low-grade inflammation in osteoarthritis. Oxidative Med Cell Longev 2018:17

Moskal JT, Capps SG (2018) Intra-articular tranexamic acid in primary total knee arthroplasty: meta-analysis. J Knee Surg 31:56-67

Murdoch AD, Grady LM, Ablett MP, Katopodi T, Meadows RS, Hardingham TE (2009) Chondrogenic differentiation of human bone marrow stem cells in transwell cultures: generation of scaffold-free cartilage. Stem Cells 25:27862796

Pap T, Korb-Pap A (2015) Cartilage damage in osteoarthritis and rheumatoid arthritis - two unequal siblings. Nat Rev Rheumatol 11:606

Parker JD, Lim KS, Kieser DC, Woodfield TBF, Hooper GJ (2018) Is tranexamic acid toxic to articular cartilage when administered topically? Bone Joint J 100-B: 404-412

Reed MR, Woolley LT (2015) Uses of tranexamic acid. Continuing Education in Anaesthesia Critical Care \& Pain 15:32-37

Schulze-Tanzil G (2009) Activation and dedifferentiation of chondrocytes: implications in cartilage injury and repair. Ann Anat 191:325-338

Solchaga LA, Penick KJ, Welter JF (2011) Chondrogenic differentiation of bone marrow-derived mesenchymal stem cells: tips and tricks. In: Mesenchymal stem cell assays and applications. Humana Press, New York

Srebro D, Vuckovic S, Milovanovic A, Kosutic J, Vujovic KS, Prostran M (2016) Magnesium in pain research: state of the art. Curr Med Chem 24:424

Takahashi A, de Andrés MC, Hashimoto K, Itoi E, Oreffo ROC (2015) Epigenetic regulation of interleukin-8, an inflammatory chemokine, in osteoarthritis. Osteoarthr Cartil 23:1946-1954

Tuttle JR, Ritterman SA, Cassidy DB, Anazonwu WA, Froehlich JA, Rubin LE (2014) Cost benefit analysis of topical tranexamic acid in primary total hip and knee arthroplasty. J Arthroplast 29:1512-1515

Weber M, Worlicek M, Voellner F, Woerner M, Benditz A, Weber D, Grifka J, Renkawitz T (2018) Surgical traning does not affect operative time and outcome in total knee arthroplasty. PLoS One 13:e0197850

Werthel J-D, Hatta T, Schoch B, Cofield R, Sperling JW, Elhassan BT (2017) Is previous nonarthroplasty surgery a risk factor for periprosthetic infection in primary shoulder arthroplasty? J Shoulder Elb Surg 26:635-640

\section{Submit your manuscript to a SpringerOpen ${ }^{\circ}$ journal and benefit from:}

- Convenient online submission

- Rigorous peer review

- Open access: articles freely available online

- High visibility within the field

- Retaining the copyright to your article

Submit your next manuscript at $>$ springeropen.com 